\title{
Comparison of the free volume sizes and shapes determined from crystallographic and PALS data
}

Maciej Tydda, Bożena Jasińska

\begin{abstract}
Two different classes of molecular crystals were investigated. The first group was benzenediols, which are characterized by the same chemical composition but a different organization of their crystallographic structures; all of the compounds from this group have only one kind of free volumes. The second class was represented by olanzapine, which has more complex chemical composition and two kinds of free volumes in the structure. The $o$-Ps lifetime values determined from positron annihilation lifetime spectroscopy (PALS) measurements agree quite well with those calculated for sizes found from crystallographic data for benzenediols (agreement within $10 \%$ of the lifetime values). For olanzapine, a good agreement is observed in the case of cuboidal free volumes, while for the other kind of void, the agreement is less satisfactory. Positronium diffusion coefficient determined from $o$-Ps redistribution in olanzapine agrees with these found for polymers.
\end{abstract}

Key words: benzenediols $\bullet$ free volume $\bullet$ molecular crystals olanzapine $\bullet$ positron annihilation $\bullet$ positronium - positronium diffusion coefficient

M. Tydda ${ }^{\bowtie}$, B. Jasińska

Department of Nuclear Methods,

Institute of Physics,

Maria Curie-Sklodowska University,

1 M. Curie-Skłodowskiej Sq., 20-031 Lublin, Poland,

E-mail: Maciej.Tydda@poczta.umcs.lublin.pl

Received: 9 July 2015

Accepted: 28 August 2015

\section{Introduction}

Positron annihilation lifetime spectroscopy (PALS) is commonly used in the investigations of the material porosity. At the beginning, it was used mainly to study free volumes in polymers while recently to mesoporous material investigations. Such technique can be successfully applied, as the mean lifetime value of ortho-positronium ( $O$-Ps) can be correlated with the size of a cavity in which this pseudo-atom annihilates. This relationship was developed for spherical voids by Tao [1] and Eldrup et al. [2]:

$$
\tau=\frac{1}{\lambda_{b}}\left[1-\frac{R}{R+\Delta}+\frac{1}{2 \pi} \sin \left(\frac{2 n R}{R+\Delta}\right)\right]^{-1}
$$

where $\lambda_{b}$ is decay constant of $o$-Ps in a 'bulk' material and equal to $2 \mathrm{~ns}^{-1}$. It was stated, however, that in many kind of materials, free volumes are rather nonspherical and some modification of the shape is needed [3, 4]. Pores in the mesoporous media are rather elongated, and cylinders better reflects their shape $[5,6]$. Relation for ortho-positronium lifetime in cuboidal free volume with the side length of $a_{i}$ is as follows:

(2) $\lambda_{p o}=\lambda_{b}\left[1-\prod_{i-3}^{3}\left(\frac{a_{i}}{a_{i}+2 \Delta}+\frac{1}{\pi} \sin \left(\frac{a_{i} \pi}{a_{i}+2 \Delta}\right)\right)\right]$.

Sizes of the pores received from PALS measurements are usually compared to those determined 
by small angle X-ray scattering (SAXS) or liquid nitrogen (LN) adsorption/desorption techniques, which are treated in material science as a porosimetric ones. However, they are limited to the size of above $1 \mathrm{~nm}$. In the case of small voids existed in polymers, for example, they cannot be applied. The only way to verify the lifetime values from experiment is to compare them to values obtained for real sizes of free spaces present in the material, determined from crystallographic data. The kind of material which can be treated as a good object for proposed verification are organic crystals.

The $o$-Ps intensity is the next parameter determined during positron lifetime spectra processing. It is correlated with positronium formation probability and trapping. In the simplest case, it is connected to the free volume amount in the material, sometimes to their number $[7,8]$. In complicated inner structures, a few components ascribed to $o$-Ps decay in a few cavity types can be found in the spectrum. The $o$-Ps fission into two components was reported for the first time by Brandt and Paulin [9] in powder investigation. In the material containing small voids and pores, fraction of $o$-Ps atoms annihilating in small voids can be calculated from the following equation $[9,10]$ :

$$
\Phi_{v}=1-\frac{3}{2} \beta\left[1-\beta^{2}+\left(1+\beta^{2}\right) \exp (-2 / \beta)\right]
$$

where $\beta=\left(\Delta \tau_{v}\right)^{1 / 2} / R, R$ is the small void radius, $\Delta$ is the diffusion coefficient, and $\tau_{v}$ is the lifetime value in small voids. The same fraction can be determined from $o$-Ps intensities: $I_{v} /\left(I_{v}+I_{p}\right)$, where $v$ and $p$ denote intensity of $o$-Ps in small voids and pores, respectively.

To study proposed problem of verification of the agreement between mean lifetime values determined from PALS measurements and lifetime values calculated from crystallographic data, three isomers of benzenediols and a stable form of olanzapine were chosen. The last crystal was also used to investigate the redistribution of Ps atoms between two kinds of voids existed in the matter.

An attempt of discussion of $o$-Ps diffusion coefficient has been made in case of material with more than one kind of free volume (more than one $o$-Ps component).

\section{Experimental materials}

Two different classes of organic crystals were investigated. The first group was benzenediols, a group of three isomers of $\mathrm{C}_{6} \mathrm{H}_{4}(\mathrm{OH})_{2}$.

The first compound of the isomer group is benzene-1,2-diol, also known as catechol (Fig. 1).

The second isomer is resorcinol (benzene-1,3-diol) (Fig. 2). There are two crystallographic phases of this compound, while only $\alpha$-phase is considered stable and is a subject of investigation in this paper.

The last compound of benzenediols group is hydroquinone (benzene-1,4-diol). Its crystallographic structure is shown in Fig. 3. Free volumes in form of infinite, bended channels exist between sheets of molecules.

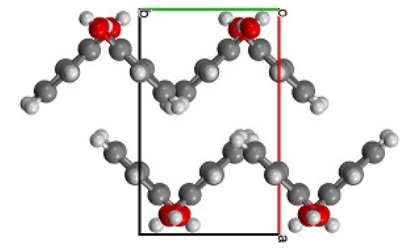<smiles>Oc1ccccc1O</smiles>

Fig. 1. Catechol: crystallographic structure (left) and molecule (right).
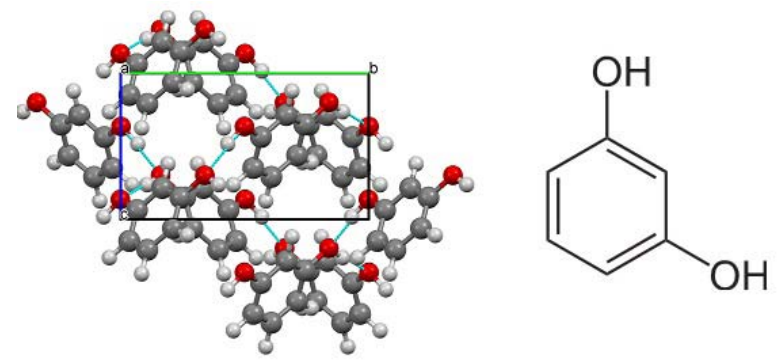

Fig. 2. Resorcinol: crystallographic structure (left) and molecule (right).

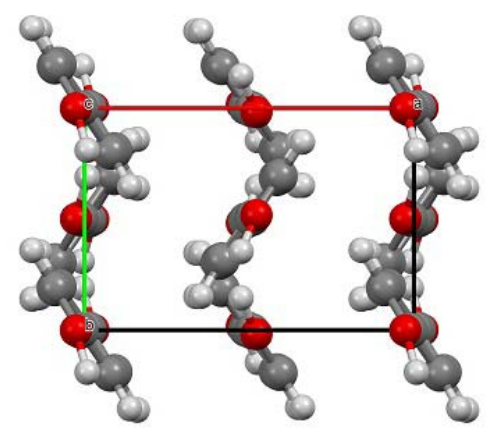<smiles>Oc1ccc(O)cc1</smiles>

Fig. 3. Hydroquinone: crystallographic structure (left) and molecule (right).

As molecules of these compounds have the same chemical composition, one can assume that there is no change in chemical interaction of positrons or positronium between each of the isomers. Observed differences in Ps formation probability could then come from accessible free volume in three investigated isomers only.

The second compound was olanzapine $\left(\mathrm{C}_{17} \mathrm{H}_{20} \mathrm{~N}_{4} \mathrm{~S}\right)$. Olanzapine has more complex chemical composition, in comparison to benzenediols, and two kinds of free volumes may be distinguished in its crystallographic structure (Fig. 4).

It is to note that olanzapine exists in many crystallographic forms but only form II is stable;
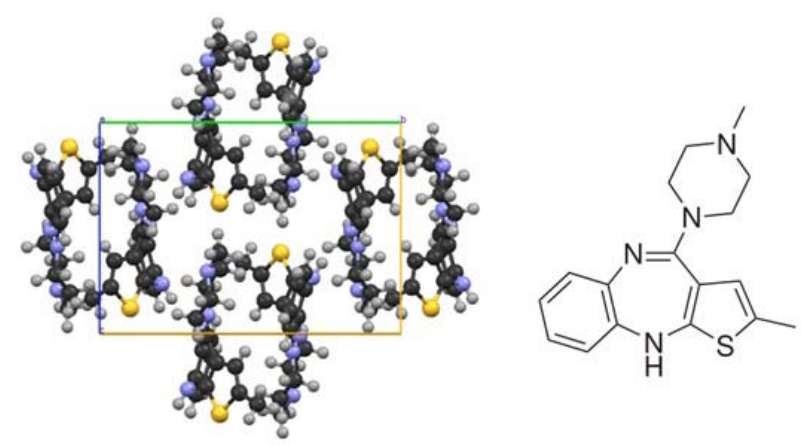

Fig. 4. Olanzapine: crystallographic structure (left) [11] and molecule (right). 
Table 1. The comparison of the $o$-Ps lifetime value for benzenediols and olanzapine received from experimental spectra processing using LT and MELT programs and calculated using Tao-Eldrup model for spherical and cuboidal shapes. The theoretical values that agrees with experiment are bolded

\begin{tabular}{|c|c|c|c|c|c|c|}
\hline \multirow{3}{*}{ Material } & \multicolumn{6}{|c|}{ Lifetime [ns] } \\
\hline & \multicolumn{3}{|c|}{ Experimental value [s] } & \multicolumn{3}{|c|}{ Theoretical values } \\
\hline & LT & & & $\begin{array}{c}\text { Sphere } \\
(\Delta=0.166 \mathrm{~nm})\end{array}$ & $\begin{array}{c}\text { Cuboid } \\
(\Delta=0.166 \mathrm{~nm})\end{array}$ & $\begin{array}{c}\text { Cuboid } \\
(\Delta=0.190 \mathrm{~nm})\end{array}$ \\
\hline$\overline{\text { Catechol }}$ & 0.86 & & & 0.68 & 1.23 & 1.09 \\
\hline Resorcinol & 0.98 & & & 0.91 & 0.62 & 0.59 \\
\hline Hydroquinone & 1.10 & 0.84 & 1.23 & 0.79 & 1.26 & 1.10 \\
\hline Olanzapine & 1.46 & 0.72 & 1.53 & 0.86 & 1.46 & 1.23 \\
\hline
\end{tabular}

therefore all investigations described in this paper refer to this one.

\section{Techniques}

PALS measurements were performed using standard 'fast-slow' coincidence spectrometer.

Time resolution of the spectrometer was about 230 ps.

All measurements were conducted at room temperature. The number of counts per spectrum was about $2 \times 10^{7}$. Spectra were analyzed using both LT and MELT procedures $[12,13]$. The LT procedure gives most probable, discrete lifetime values, while MELT algorithm provides distribution of lifetimes and averaged value for calculated distribution (not the value for highest intensity).

\section{Results and discussion}

Free volumes in crystallographic structure of catechol exist between hydrogen-bonded layers of molecules, forming zig-zag-shaped infinite channels. The cross section of this channel calculated using atomic radii of atoms is $1.85 \times 5.65 \AA$ (Fig. 1), which results in theoretical $o$-Ps lifetime of 1230 ps. It was suggested for many times that parameter $\Delta$ used in Tao-Eldrup model determined for $0.166 \mathrm{~nm}$ can change depending on the kind of materials $[3,14,15]$. Theoretical lifetime values for determined cuboid were calculated using the value of $\Delta=0.19 \mathrm{~nm}$ proposed before for elongated channels in crystals, additionally (see Table 1 ). For such $\Delta$ value, calculated $o$-Ps lifetime assuming cuboidal shape is equal to $1090 \mathrm{ps}$. However, lifetime measured in experiment is much shorter and its value is set at 863 ps as received using LT program and 960 ps from MELT deconvolution (Fig. 5a).

In the structure of resorcinol, ellipsoidal free volume with semi-axis length of $2 \AA$ and $1.5 \AA$ (Fig. 2) exists, respectively. This translates into 910-ps theoretical $o$-Ps lifetime (spherical void, $\Delta=0.166 \mathrm{~nm}$ ), while in experiment, this values are set at 980 and $960 \mathrm{ps}$ as calculated by LT and MELT programs, respectively (Fig. 5a).

In hydroquinone, channel cross section is $2.0 \times 4.8 \AA$ (Fig. 3). This results in theoretical $o$-Ps lifetime of $1260 \mathrm{ps}$ for $\Delta=0.166 \mathrm{~nm}$. If we use $\Delta=$ $0.19 \mathrm{~nm}$ similar to that in the case of cuboidal free volumes in catechol, the $o$-Ps lifetime is shortened to 1100 ps. Experimental value obtained from LT is $1100 \mathrm{ps}$. However, lifetime distribution calculated by MELT suggests an existence of two $o$-Ps components with lifetimes of 840 and $1230 \mathrm{ps}$. This is because MELT sometimes divides one wide component into two (Fig. 5a). This effect was stated and well described $[16,17]$ in literature and appears also in simulated spectra containing only one lifetime value.

As mentioned before, in crystallographic structure of olanzapine, two kinds of free volumes exist. First kind of free volume is in between molecules forming dimers and can be described as sphere with radius of about $1.4 \AA$. The second cavity is localized between dimers and have dimensions of approximately $5.3 \times 3.9 \times 3.6 \AA$ (Fig. 4). The lifetimes
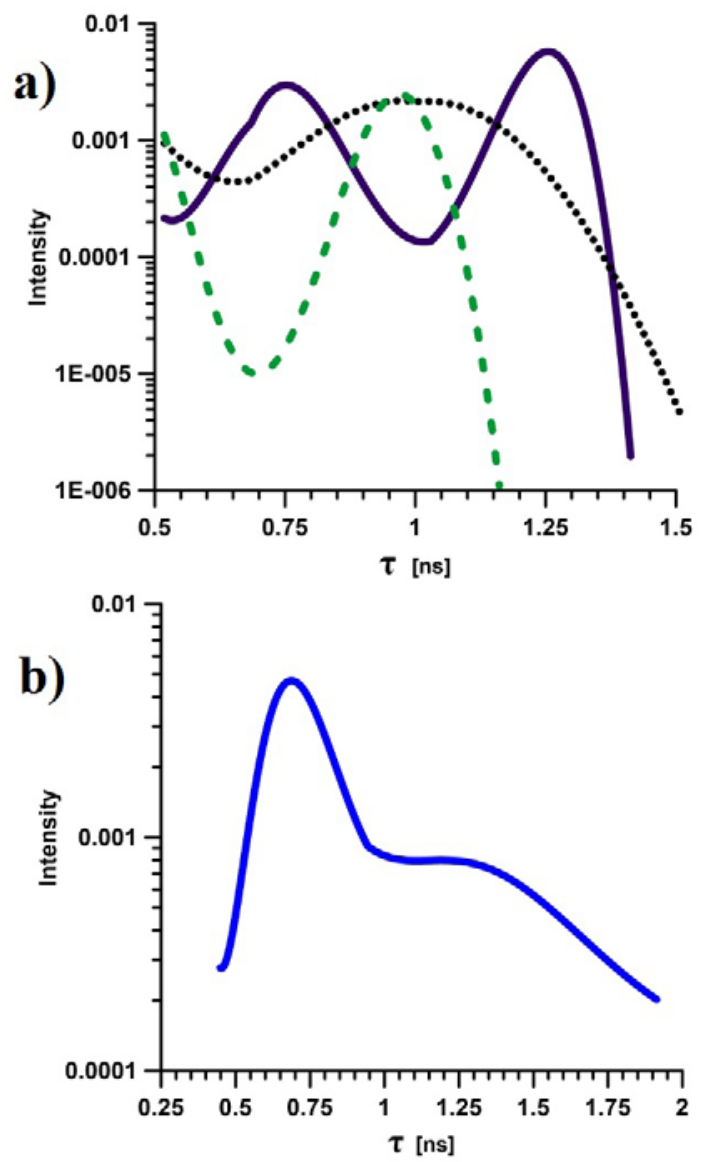

Fig. 5. The lifetime distribution of $o$-Ps components as received from MELT routine (a) in benzenediols: hydroquinone (solid line), resorcinol (dotted line), and catechol (dashed line) and (b) olanzapine. 
Table 2. Values of $o$-Ps diffusion coefficient $(D)$ calculated for several values of parameters $\beta, R$, and constant value of $\tau_{v}$

\begin{tabular}{|c|c|c|c|c|}
\hline$\beta$ & 0.115 & 1.13 & 0.115 & 1.13 \\
\hline$\overline{R[\mathrm{~nm}]}$ & 0.14 & 0.14 & 0.12 & 0.12 \\
\hline$\tau_{v}[\mathrm{~ns}]$ & 0.72 & 0.72 & 0.72 & 0.72 \\
\hline$D\left[\mathrm{~cm}^{2} / \mathrm{s}\right]$ & $0.360 \times 10^{-6}$ & $0.348 \times 10^{-4}$ & $0.265 \times 10^{-6}$ & $0.255 \times 10^{-4}$ \\
\hline
\end{tabular}

found using LT program in experiment are equal to 670 and 1460 ps and 720 and 1530 ps using MELT procedure (Fig. 5b). Shorter lifetime is ascribed to the free volume inside the dimer (spherical), while longer one to the void between dimers (cuboidal). The theoretical lifetimes calculated for both voids are 860 and 1460 ps for spherical and cuboidal free volume, respectively. In both cases, $\Delta=0.166 \mathrm{~nm}$ was used.

Results of positron lifetimes for all samples are gathered in Table 1 . Theoretical values were calculated additionally for both spherical and cuboidal shapes independently on the real shape existing in the structure. It gives a possibility to compare the experimental results to the values received using an inadequate shape. In our opinion, the best agreement between experiment and theoretical predictions was found in the case of a sphere with $\Delta=0.166$ for resorcinol, while in the case of a cuboidal shape, the better value is $\Delta=0.19 \mathrm{~nm}$, what seems to confirm the value proposed before [3]. For olanzapine, the great difference between theory and experiment is observed for smaller spherical volume existed inside a dimer. However, this void has rather more complicated shape than a sphere. The discrepancies between model predictions and experiment were observed before for short-lifetime values in the studies by Zgardzinska et al. [18].

As it was described earlier, olanzapine is a compound in which two kinds of well-defined free volumes exist in the structure. It causes $o$-Ps redistribution between them and in the spectra processing two components ascribed to $o$-Ps annihilation were found. Discussion of Ps trapping in various kind of cavities seems to be very important. In the papers $[9$, 10], one described outdiffusion of Ps from small to large voids. However, in vast group of materials, two kind of angstroms size voids exists. Question is the kind of process leading to Ps redistribution between them. We suppose that according to the blob model proposed by Stepanov [19], pre-positronium created in the blob can move in same limited range in the material before being trapped in the free volume. Probability of trapping in respective size of void can then be the function of voids size (radius, volume, or surface area).

Intensities of both $o$-Ps components in olanzapine reach $34 \%$ and $7 \%$. Fraction of $o$-Ps annihilating in the smaller pores is then equal to 0.83 . If we solve the equation for $\Phi_{v}=0.83$, we obtain $\beta_{1}=0.115$ or $\beta_{2}=1.13$. Positronium diffusion coefficient may be calculated from the relationship $D=(\beta R)^{2} / \tau_{v}$. Diffusion coefficient values calculated for two values of $R$ and lifetime value from experiment are shown in Table 2.

As a solution, we received two values of Ps diffusion coefficient. A value calculated for $\beta=0.115$ is one order of magnitude smaller than that for amorphous polymers [20], while value for $\beta=1.13$ are same order of magnitude as for $\mathrm{SiO}_{2}$ in [9]. Despite of some discrepancies between experimental values and crystallographic data presented in the paper, diffusion coefficient value agree with these calculated before for dense solids.

\section{Conclusions}

Analysis of the PAL spectra can provide information about approximated shapes and sizes of the free volumes based on Tao-Eldrup model with its modifications. Agreement between theoretical values calculated from the model stays within $10 \%$ compared to values obtained from the experiment. It is interesting that we obtain a better agreement between the experiment and the model if we calculated the lifetime values from the model using parameter $\Delta=$ $0.166 \mathrm{~nm}$ for sphere, while $\Delta=0.19 \mathrm{~nm}$ seem better for cuboid. It is then possible that the $\Delta$ value, which represents Ps wave function penetration into matter, is also influenced by the cavity shape, not only by the kind of material (electron density). Additional technique of spectra deconvolution (MELT) allows to see cavity deformations from wider lifetime distributions that can help in shape determination; the wider the distribution, the more deformed is the void.

The Ps diffusion coefficient determined from $o$-Ps intensities in two kind of voids, agree with these determined for doped polymers containing only one kind of voids. It suggests that model of $o$-Ps diffusion, proposed elsewhere for porous material, can be probably applied even in the case of small voids.

\section{References}

1. Tao, S. J. (1972). Positronium annihilation in molecular substances. J. Chem. Phys., 56, 5499-5510. DOI: 10.1063/1.1677067.

2. Eldrup, M., Lightbody, D., \& Sherwood, J. N. (1981). The temperature dependence of positron lifetimes in solid pivalic acid. Chem. Phys., 63, 51-58. DOI: 10.1016/0301-0104(81)80307-2.

3. Jasińska, B., Kozioł, A. E., \& Goworek, T. (1996). Ortho-positronium lifetimes in nonspherical voids. J. Radioanal. Nucl. Chem., 210(2), 617-623. DOI: 10.1007/BF02056403.

4. Jasińska, B., Kozioł, A. E., \& Goworek, T. (1999). Void shapes and o-Ps lifetime in molecular crystals. Acta Phys. Pol. A, 95, 557-561.

5. Goworek, T., Ciesielski, K., Jasińska, B., \& Wawryszczuk, J. (1997). Positronium in large voids. Silicagel. Chem. Phys. Lett., 272, 91-95. DOI: 10.1016/S00092614(97)00504-6.

6. Ciesielski, K., Dawidowicz, A., Goworek, T., Jasińska, B., \& Wawryszczuk, J. (1998). Positronium lifetimes 
in porous Vycor glass. Chem. Phys. Lett., 289(1/2), 41-45. DOI: 10.1016/S0009-2614(98)00416-3.

7. Kobayashi, Y., Zheng, W., Meyer, E. F., McGervey, J. D., Jamieson, A. M., \& Simha, R. (1989). Free volume and physical aging of poly (vinyl acetate) studied by positron annihilation. Macromolecules, 22(5), 2302-2306. DOI: 10.1021/ma00195a052.

8. Dlubek, G., Pionteck, J., Sniegocka, M., Hassan, E. M., \& Krause-Rehberg, R. (2007). Temperature and pressure dependence of the free volume in the perfluorinated polymer glass CYTOP: A positron lifetime and pressure-volume-temperature study. J. Polym. Sci. Pt. B-Pol. Phys., 45(18), 2519-2534. DOI: 10.1002/ polb.21248.

9. Brandt, W., \& Paulin, R. (1968). Positronium diffusion in solids. Phys. Rev. Lett., 21, 193-195. DOI: 10.1103/PhysRevLett.21.193.

10. Venkateswaran, K., Cheng, K. L., \& Jean, Y. C. (1984). Application of positron annihilation to study the surface properties of porous resins. J. Phys. Chem., 88, 2465-2469. DOI: 10.1021/j150656a010.

11. Tydda, M., Jasińska, B., Kozioł, A. E., \& Wawrzycka-Gorczyca, I. (2013). Modification of the crystallographic structure of olanzapine during solvation by PALS and X-ray diffraction methods. Mater. Sci. Forum, 733, 92-95.

12. Kansy, J. (1996). Microcomputer program for analysis of positron lifetime spectra. Nucl. Instrum. Methods Phys. Res. Sect. A-Accel. Spectrom. Dect. Assoc. Equip., 374, 235-244. DOI: 10.1016/01689002(96)00075-7.

13. Shukla, A., Peter, M., \& Hoffmann, L. (1993). Analysis of positron lifetime spectra using quantified maximum entropy and a general linear filter. $\mathrm{Nucl}$. Instrum. Methods Phys. Res. Sect. A-Accel. Dect.
Assoc. Equip., 335, 310-317. DOI: 10.1016/01689002(93)90286-Q.

14. Jasińska, B., \& Dawidowicz, A. L. (2003). Pore size determination in Vycor glass. Radiat. Phys. Chem., 68, 531-534. DOI: 10.1016/S0969-806X(03)00224-X

15. Gidley, D. W., Frieze, W. E., Dull, T. L., Yee, A. F., Ryan, E. T., \& Ho, H. M. (1999). Positronium annihilation in mesoporous thin films. Phys. Rev. B, 60(8), 5157-5160. DOI: 10.1103/PhysRevB.60.R5157.

16. Dlubek, G., Eichler, S., Hubner, Ch., \& Nagel, Ch. (1999). Does the MELT program accurately reveal the lifetime distribution in polymers? Phys. Status Solidi A, 174, 313-325. DOI: 10.1002/ (SICI) 1521-396X(199908) 174:2<313::AIDPSSA313 > 3.3.CO;2-U.

17. Dlubek, G., Hubner, Ch., \& Eichler, S. (1998). Do the CONTIN or the MELT programs accurately reveal the o-Ps lifetime distribution in polymers? Analysis of experimental lifetime spectra of amorphous polymers. Nucl. Instrum. Methods Phys. Res. Sect. B-Beam Interact. Mater. Atoms, 142, 191-202. DOI: 10.1016/ S0168-583X(98)00265-1.

18. Zgardzinska, B. (2015). The size of smallest subnanometric voids estimated by positron annihilation method. Correction to the Tao-Eldrup model. Chem. Phys. Lett., 62, 20-22. DOI: 10.1016/j.cplett.2015.01.021.

19. Stepanov, S., \& Byakov, V. (2003). Physical and radiation chemistry of positron and positronium. In Y. C. Jean, P. Mellon, \& D. M. Schradder (Eds.), Principles and applications of positron and positronium chemistry (pp. 117-148). Singapore: World Scientific. DOI: 10.1142/9789812775610_0005.

20. Hirata, K., Kobayashi, Y., \& Ujihira, Y. (1996). Diffusion coefficients of positronium in amorphous polymers. J. Chem. Soc., Faraday Trans., 92, 985-988. 